\title{
International harmonization of bioanalytical guidance
}

\author{
"Multiple guidance and regulations create additional burden to performing quality science in a global work place and create \\ potential conflicts with each other in substance and/or interpretation."
}

Science and regulations both have their place and importance in bioanalysis. We may not know the exact timing of their origin, but bioanalysts know that they have co-existed for many years, and that both have progressed with controlled but constant transformation. One has influenced the other but, by and large, science and regulations have had their individual developmental pathways.

When we talk of scientific development, bioanalysis has seen some excellent progress in multiple areas, for example speed, specificity, sensitivity, sample size and data volume. As both our technologies and knowledge of the implications of endogenous sample matrices have improved, we can now develop highly sensitive and specific bioanalytical methods for analysis of drugs and metabolites in complex matrices in a matter of days using LC-MS/MS, whereas it used to take months to develop even a method with several orders of magnitude lower sensitivity. LC-MS/MS has revolutionized the bioanalytical field, just as the internet has revolutionized the information field. We can no longer imagine getting information without internet searches. Similarly, we cannot imagine developing bioanalytical methods of adequate sensitivity and specificity using LC-UV. Equally impactful has been the explosive growth in immunoassay technology for biologic therapeutics with its improvements in sample volume requirements, specificity, multiplexing capabilities and speed. Automation, speed of analysis, data capture and archive have all but replaced the classical approaches of bioanalysis.

Parallel to this dramatic scientific progress in the bioanalytical field, rules and regulations have also been revised or new ones have been introduced. However, the regulatory rules and guidance revision/additions have taken their own course. In the old days, we had regulations, but no specific guidance on how to perform the bioanalytical work. The regulations required that bioanalytical methods should be demonstrated to be accurate and of sufficient sensitivity to measure drugs and metabolites with appropriate precision. It was left to the scientist to develop and present sufficient scientific evidence that the bioanalytical work followed and conformed to the regulations. Then came the first joint conference/workshop of scientists and regulators in 1990, popularly known as the 'Crystal City Bioanalytical Conference.' The workshop had global participation and developed essential parameters for bioanalytical methods validation. The workshop recommendations were documented in a scientific White Paper [1].

"...bioanalysis has seen some excellent progress in multiple areas, for example speed, specificity, sensitivity, sample size and data volume."

The first Crystal City White Paper provided detailed procedures and requirements on how to validate and use the bioanalytical methods to measure drug and metabolite concentrations in biological matrices. The requirements, procedures and interpretations were equally acceptable to both the practitioners and regulators of bioanalysis. The paper rapidly grew in popularity around the world, as it provided a harmonized framework for bioanalytical processes to meet the expectations of regulatory review and inspections. It minimized individual interpretations or long explanations on how a scientist would quantitatively determine parameters of an assay to measure its sensitivity, accuracy and precision. Scientists loved it because it established a clear set of expectations and they did not have to individually devise and develop ways to fulfill the regulatory requirements for bioanalysis. A decade later there was another 'Crystal City Bioanalytical Conference/Workshop’ and

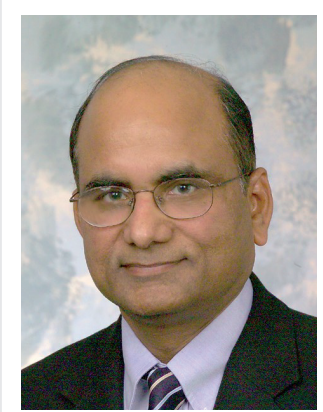

Surendra K Bansal

Author for correspondence Hoffmann-La Roche Inc., Nutley, NJ, USA

E-mail: surendra.bansal@ roche.com

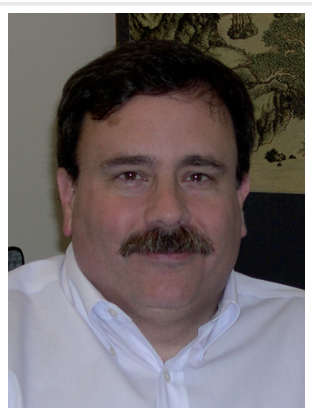

Mark Arnold

Bristol-Myers Squibb

Company, Princeton, NJ, USA

E-mail: mark.arnold@

bms.com

Authors continued overleaf... 


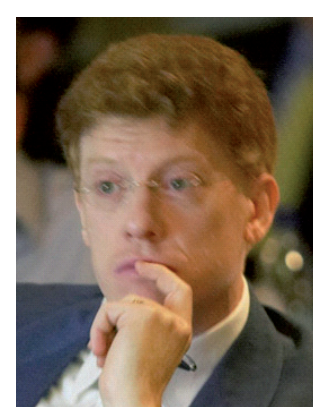

Fabio Garofolo

Algorithme Pharma Inc., Laval, QC, Canada

E-mail: fgarofolo@ algopharm.com the US FDA issued regulatory guidance on bioanalysis [2]. It was issued as guidance, and not as new regulation (law). Not following the guidance is considered acceptable, as long as you justify the alternative approach. Questions lingered with bioanalysts on how to make sure that the alternative approach(es) would be considered equivalent to providing the essential parameters to prove the accuracy, precision and sensitivity of the assay. Therefore, bioanalysts started to follow the guidance and subsequent White Papers to the tee.

Bioanalytical science for both large and small molecules continued progression at an unprecedented rate. Further conferences and workshops with regulators' participation were held around the world, and the FDA guidance was supplemented by additional White Papers. In this rapidly developing era of bioanalysis, it is no longer sufficient to perform the work according to the FDA guidance; one must also conform to the new requirements provided in White Papers. Recently, the European Medicines Agency (EMA) issued a draft guideline [3] on the validation of bioanalytical methods. This draft guideline is very similar to the FDA Guidance and Crystal City conference reports, and keeps the legacy of bioanalytical practice that has been in use for the last two decades. However, it does contain a few differences. The FDA is also revising its 2001 guidance [2]. It is very reassuring to see that the regulatory agencies are providing guidance that is changing with the continuous evolution of bioanalytical science. It is in the best interests of everyone to adopt simple guidance whose interpretations would be equally acceptable to both the practitioners and regulators of bioanalysis.

In this global economy, many pharmaceutical companies and contract research organizations generate bioanalytical data that are submitted to regulatory agencies around the world. Studies are conducted globally with the samples analyzed in one country and the results submitted to regulatory agencies in other countries. Multiple guidance and regulations create additional burden to performing quality science in a global work place and create potential conflicts with each other in substance and/ or interpretation. It would be of great benefit to the industry and regulatory agencies if bioanalytical guidelines were harmonized among major regulatory agencies such as the EMA, FDA, Japanese MHLW, Canadian HC-TPD, Brazilian ANVISA and Australian TGA. Does a system exist for creating a joint regulatory guidance that could be accepted globally? We are not aware of any such system or document of agreement that would be universally acceptable to the regulatory agencies around the world. So what is the solution? One solution could be going back to the 1990s, when there were regulations for performing bioanalytical work but the scientific guidance was provided through White Papers accepted by both bioanalysts and regulatory agencies. As the scientific development continues at a rapid pace, this hybrid process of the 1990s may provide a solution where good science and compliance would co-exist and thrive to provide necessary regulatory data to drive new medicines to patients globally. As was neccessary in the past, this system would require a commitment from regulatory agencies and practitioners of bioanalysis to join forces in extended global bioanalytical workshops. Already, the major conferences have global participation. All we need is an increased participation of representatives from more regulatory agencies. Such a 'World Congress of Bioanalysis' equivalent to an extended 'Crystal City Bioanalytical Conference/Workshop' could tie up the loose ends of bioanalytical differences by providing consensus reports acceptable to both the practitioners and regulators of bioanalysis.

\section{"Ultimately, the patients using pharmaceutical products would be the winners through the consistent generation of high-quality data and faster and more consistent review practices."}

Additionally, or alternatively, if OECD guidelines or consensus documents could be accepted by worldwide regulatory agencies, the bioanalytical guidance created under the auspices of the OECD with active participation/ comments from the global bioanalytical community, would achieve the desired global harmonization. Keeping pace with high-quality science, harmonized scientific guidance would be the key to providing uniform procedures meeting global regulatory expectations. To the extent that such a document would foster improved scientific quality and compliance, the results would benefit both the regulatory agencies and the pharmaceutical industry. Ultimately, the patients using pharmaceutical products would be the winners through the consistent generation of high-quality data and faster and more consistent review practices. 
Financial \& competing interests disclosure

The authors have no relevant affiliations or financial involvement with any organization or entity with a financial interest in or financial conflict with the subject matter or materials discussed in the manuscript. This includes employment, consultancies, honoraria, stock ownership or options, expert testimony, grants or patents received or pending, or royalties.

No writing assistance was utilized in the production of this manuscript.

\section{Bibliography}

1 Shah VP, Midha KK, Dighe S et al. Analytical methods validation: bioavailability, bioequivalence and pharmacokinetic studies. Phar. Res. 9(4), 588-592 (1992).
2 US FDA. Guidance for industry: bioanalytical method validation. US Department of Health and Human Services, Food and Drug Administration, Center for Drug Evaluation and Research (2001).
3 European Medicines Agency, Committee for Medicinal Products for Human Use. Guideline on Validation of Bioanalytical Methods (Draft), EMA/CHMP/ EWP/192217/2009. 